\title{
Individuelle Dermalfiller-Behandlung bei weniger Produktüberschuss
}

Mit Juvéderm ${ }^{\circledast}$ Volift $^{\mathrm{Tm}}$ Retouch erweitert Allergan seine Vycross ${ }^{\mathrm{rm}}$ Kollektion, mit der sich sichtbare und lang anhaltende Resultate für bis zu 18 Monate erzielen lassen. Juvéderm ${ }^{\oplus}$ Volift $^{\mathrm{T} m}$ mit Lidocaine ist ein vielfältiger Dermalfiller und gut für Folgebehandlungen geeignet. Die Einheit mit 0,55 $\mathrm{ml}$ wird angeboten, damit Ärzte das Ergebnis der Erstbehandlung mit Dermalfillern verfeinern und auffrischen können sowie gleichzeitig überschüssige, ungenutzte Produktmengen reduzieren können.

Untersuchungen zeigen immer wieder, wie wichtig Patienten ein möglichst natürliches Ergebnis ist. Sie wünschen sich eine Behandlung, die ihren ursprünglichen Gesichtsausdruck erhält. Um dieses Ergebnis zu erzielen, ist nicht nur ein eingehendes Beratungsgespräch, sondern auch eine subtile, maßgeschneiderte Behandlung wichtig. Das bedeutet, dass insbesondere bei Folgeterminen und Nachbehandlungen die Standard-Menge von $1 \mathrm{ml}$ pro Spritze mitunter nicht mehr benötigt wird. Die Einheit mit 0,55 $\mathrm{ml} \mathrm{re-}$ duziert die Wahrscheinlichkeit überschüssiger Produktmengen und ist auf die Bedürfnisse von Ärzten abgestimmt, die ihren Patienten einen individuellen Behandlungsplan bieten wollen.

Der Dermalfiller kann vielfältig angewendet werden, um entsprechende Resultate beim Auffüllen von mittleren und tiefen Gesichtsfalten, beim Wiederaufbau von Volumen und bei der Verbesserung der Gesichtskontur zu erzielen oder zu erhalten.

„Die große Mehrheit meiner Patienten will ein natürlich wirkendes Ergebnis", sagte Dr. med. Daniela Greiner, Fachärztin für Dermatologie mit eigener Praxis in Ober-Ursel bei Frankfurt. „Ich erkläre ihnen, dass ich gezielt geringe Mengen eines Dermalfillers einsetze, um einen subtilen Lifting-Effekt und eine Verbesserung der Gesichtskontur zu erzielen. Gerade weil diese Art der Behandlung

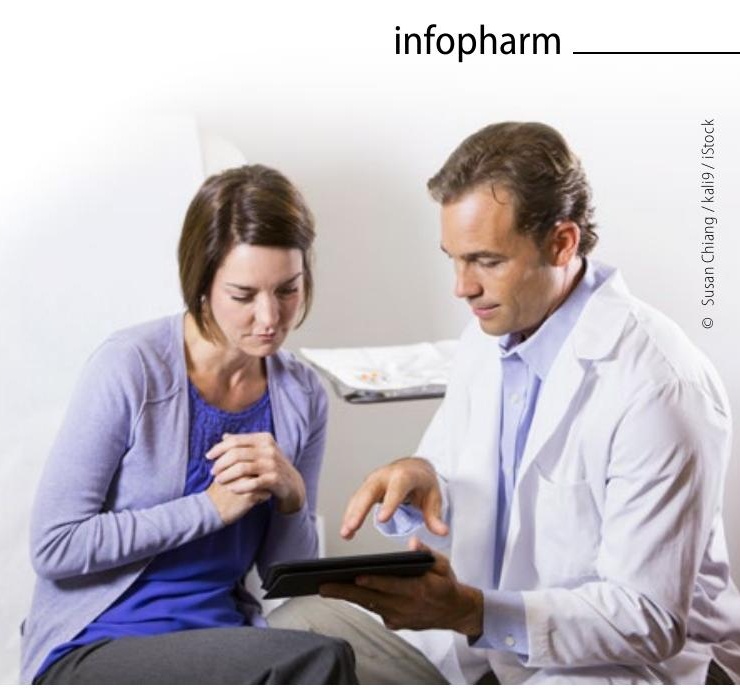

Für ein optimales Ergebnis zur Gesichtsverjüngung ist das ausführliche Beratungsgespräch unabdingbar.

immer gefragter wird, freue ich mich, dass ich Juvéderm ${ }^{\oplus}$ Volift $^{\mathrm{Tn}}$ Retouch künftig bei meinen Patienten nutzen kann, um ansprechend aussehende Resultate zu erzielen. Dies gilt speziell für Folgebehandlungen, bei denen Patienten häufig eine kleine Auffrischung benötigen, da ich auf die Menge zurückgreifen kann, die ich wirklich brauche, und am Ende kein ungenutztes Produkt übrigbleibt, was natürlich Kosten reduziert.“

Nach Informationen von Pharm-Allergan

\section{Leidensdruck der Betroffenen wird häufig unterschätzt}

Hyperhidrose ist nicht nur ein dermatologisches Problem. Übermäßiges Schwitzen ist auch eine häufige Begleiterscheinung insbesondere bei Patientinnen im Klimakterium oder mit gynäkologischen Tumoren. Als hormonfreie Therapiealternative bietet sich das orale Anticholinergikum Methantheliniumbromid an. Sekundäre Formen der Hyperhidrose können auftreten als Symptom von Systemerkrankungen wie Lymphomen, bei neurologischen Störungen wie Morbus Parkinson oder bei endokrinologischen Krankheiten wie Diabetes. „Die komorbide, sekundäre Hyperhidrose verstärkt den Leidensdruck der Grunderkrankung und hat daher einen erheblichen zusätzlichen Einfluss auf das körperliche, psychische und soziale Leben der Betroffe- nen“, erklärte Dr. med. Petra Sandow, Fachärztin für Allgemeinmedizin in Berlin.

In der gynäkologischen Praxis tritt die sekundäre Hyperhidrose meist als Symptom des Klimakteriums in Erscheinung. Ebenso kann das Unterbinden der körpereigenen Östrogenproduktion im Rahmen einer adjuvanten Therapie des Mammakarzinoms zu einer sekundären Hyperhidrose führen. „Schweißfluss ist besonders bei Frauen mit einem starken Schamgefühl behaftet. Das Leiden wird verschwiegen und der Kontakt zu Mitmenschen gemieden“, so die Erfahrung von Dr. med. Matthias Krick, Facharzt für Gynäkologie in Moers. Er empfahl, die Patientin aktiv auf übermäßiges Schwitzen anzusprechen.
Die Hormonersatztherapie (HET) zur Behandlung der vasomotorischen Symptomatik wird kontrovers diskutiert, zudem ist sie beispielsweise bei Vorliegen eines Mamma- oder Endometriumkarzinoms kontraindiziert. In diesen Fällen ist Methantheliniumbromid (Vagantin ${ }^{\circledast}$ ) eine hormonfreie Therapieoption. Auch bei Patientinnen, bei denen eine Behandlung mit Phytoöstrogenen nicht zum gewünschten Erfolg führt, kann dieses orale Anticholinergikum eine Alternative sein. Die Substanz überwindet aufgrund seiner hydrophilen Eigenschaften kaum die BlutHirn-Schranke, so dass keine zentralnervösen Nebenwirkungen zu erwarten sind. Durch den schnellen Wirkeintritt innerhalb von 30 Minuten und der Wirkdauer von etwa 6 Stunden kann Methantheliniumbromid bei Bedarf oder bei dreimal täglicher Einnahme dauerhaft eingesetzt werden.

Gudrun Girrbach

Pressegespräch „Der Hyperhidrose-Patient in der täglichen Praxis - Erkennen und behandeln"; Düsseldorf, 17.04.2015; Veranstalter: Riemser Pharma 